\title{
PROBABILITY DISTRIBUTIONS OF EXTREMES OF SELF-SIMILAR GAUSSIAN RANDOM FIELDS
}

\author{
YURIY KOZACHENKO AND VitALII MAKOGIN
}

\begin{abstract}
We have obtained some upper bounds for probability distributions of extremes of a self-similar Gaussian random field with stationary rectangular increments, which is defined on a compact space. In the paper we also present the probability distributions of extremes for normalized self-similar Gaussian random fields with stationary rectangular increments defined on $\mathbb{R}_{+}^{2}$. In our work we have used the techniques developed for self-similar fields and based on the classical series analysis of the supremum distribution for Gaussian fields.
\end{abstract}

Mathematics subject classification (2010): 60E05, 60E15.

Keywords and phrases: Distribution of extremes, self-similar random field, finite dimensional distributions, fractional Brownian sheet.

\section{REFERENCES}

[1] R. J. ADLER, An introduction to continuity, extrema, and related topics for general Gaussian processes, Lecture Notes-Monograph Series, i-155, (1990).

[2] A. Ayache, S. Leger, M. Pontier, Drap Brownien fractionnaire, Potential Anal. 17 (1), 31-43 (2002).

[3] D. Benson, M. M. Meerschaert, B. BäUmer, H.-P. Scheffler, Aquifer operator scaling and the effect on solute mixing and dispersion, Water Resour. Res. 42, 1-18 (2006).

[4] A. Bonami, A. Estrade, Anisotropic analysis of some Gaussian models, J. Fourier Anal. Appl. 9, 215-236 (2003).

[5] S. DaVIES, P. HALL, Fractal analysis of surface roughness by using spatial data (with discussion), J. R. Stat. Soc. B 61, 3-37 (1999).

[6] V. A. Dmitrovs KiI, On the maximum distribution and local properties of the realizations of preGaussian fields, Teor. Ver. Mat. Statist. 25, 154-164 (1981).

[7] P. Embrechts, M. Maejima, Selfsimilar Processes, Princeton University Press (2001).

[8] M. G. Genton, O. Perrin, M. S. TAQQU, Self-similarity and Lamperti transformation for random fields, Stochastic Models 23, 397-411 (2007).

[9] J. HÜSler, V. PIterbarg, Extremes of a certain class of Gaussian processes, Stochastic Processes and their Applications 83 (2), 257-271 (1999).

[10] A. Kamont, On the fractional anisotropic Wiener field, Probability and Mathematical StatisticsPWN 16 (1), 85-98 (1995).

[11] Y. Kozachenko, A. Melnikov, Y. Mishura, On drift parameter estimation in models with fractional Brownian motion, arXiv:1112.2330v1 [math.PR].

[12] Y. Kozachenko, O. Vasylyk, R. Yamnenko, On the probability of exceeding some curve by $\phi$-sub-Gaussian random process, Theory Stoch. Process. 9 (3-4), 70-80 (2003).

[13] W. Z. LU, X. K. WANG, Evolving trend and self-similarity of ozone pollution in central Hong Kong ambient during 1984-2002, The Science of the Total Environment 357, 160-168 (2006).

[14] A. Morata, M. L. Martin, M. Y. Luna, F. Valero, Self-similarity patterns of precipitation in the Iberian Peninsula, Journal of Theoretical and Applied Climatology 85, 41-59 (2006).

[15] V. I. Piterbarg, Asymptotic Methods in the Theory of Gaussian Processes and Fields, Amer. Math. Soc. (2012). 
[16] G. Samorodnitsky, M. S. TAqQU, Stable Non-Gaussian Random Processes: Stochastic Models with Infinite Variance, Chapman \& Hall (1994).

[17] O. VASYLyK, Y. KoZAChenKo, R. YAMnEnKo, $\varphi$-sub-Gaussian random processes, Kyiv University, Kyiv, (2008) (in Ukrainian).

[18] Y. XIAO, Sample path properties of anisotropic Gaussian random fields, A minicourse on stochastic partial differential equations, Springer Berlin Heidelberg, 145-212 (2009). 